Title: IgE-response to peanut allergens is related with age and onset of disease in paediatric population

Authors: Garcia-Blanca $\mathrm{A}^{1}$, MD; Aranda $\mathrm{A}^{2}$, PhD; Blanca-Lopez N $\mathrm{N}^{1}$, MD, PhD; Perez $\mathrm{D}^{1}$, MD; Gomez $\mathrm{F}^{3}$, MD, PhD; Mayorga $\mathrm{C}^{2}$, $\mathrm{PhD}$; Torres $\mathrm{MJ}^{3}$, MD, PhD; Diaz-Perales $\mathrm{A}^{4}, \mathrm{PhD}$; Perkins $\mathrm{JR}^{2}$, PhD; Villalba $\mathrm{M}^{5}$, PhD; Blanca $\mathrm{M}^{3}$, MD; PhD; Canto $\mathrm{G}^{1}, \mathrm{MD}$, PhD.

Affiliation: ${ }^{1}$ Allergy Service, Infanta Leonor Hospital, Madrid, Spain; ${ }^{2}$ Research Laboratory, IBIMA, Regional University Hospital of Malaga, UMA, Malaga, Spain; ${ }^{3}$ Allergy UGC, IBIMA, Regional University Hospital of Malaga, UMA, Malaga, Spain; ${ }^{4}$ Plant Biotechnology Institute (UPM-INIA), Madrid, Spain; ${ }^{5}$ Biochemistry and Molecular Biology Department, University Complutense Madrid, Spain.

Corresponding author and Address for reprint requests: Ana Aranda Guerrero. Research Laboratory, IBIMA, Regional University Hospital of Malaga, UMA, Plaza del Hospital Civil s/n, pabellón 5, sótano. 29009 Malaga, Spain. TEL: +34 951290346. FAX: +34 951290302. E-mail: anandaguerrero@gmail.com

Short title: Response to peanut allergens in children and adolescents.

Abbreviations: SSP, seed storage proteins; LTP, lipid transfer proteins; SPT, skin prick test; PR-10, pathogenesis related-10; double-blind-placebo-controlled food challenge (DBPCFC); OAS, oral allergy syndrome.

Key Words: Food allergy, peanut, peach, allergens, sensitization, LTP, SSP

Funding Source: The study was funded by the FIS-Thematic Networks and Cooperative Research Centres: RIRAAF (RD07/0064 and RD012/0013), Junta de Andalucía (CTS-7433), and ISCIII (PI12/02481).

Conflict of Interest: None of the authors has any conflict of interest.

Financial Disclosure: None author received money for the present study. Research is part of their daily activities. All authors had full access to all the data (including statistical reports) and can take responsibility for the integrity of the data and the accuracy of the data analysis.

What's Known on This Subject: The molecular response to vegetal food allergens varies with age, with allergens from food and inhalants playing an important role. This is important when evaluating allergic reactions to peanut.

What This Study Adds: Peanut allergy patients show different recognition patterns to peanut allergens according to age. Ara h 2 sensitization is more frequent at an early age, changing to a predominance of Ara h 9 in older patients. 


\section{Contributor's Statement:}

Garcia-Blanca A: MD Garcia-Blanca collected patient's sera and analyzed the data Aranda A: Dr. Aranda performed the experiments, wrote the manuscript, coordinated data collection approved the final manuscript.

Blanca-Lopez N and Perez D: Dr. Blanca-Lopez and Perez collected patient's sera.

Gomez F: Dr. Gomez analyzed the data

Mayorga $C$ and Torres MJ: Dr. Mayorga and Dr. Torres conceived and designed the experiments and contributed reagents/materials/analysis tools

Diaz-Perales A and Villalba $M$ : Dr. Diaz-Perales and Dr. Villalba revised the manuscript

Perkins JR: Dr. Perkins wrote the manuscript

Blanca $M$ and Canto G: Dr. Blanca and Dr. Canto coordinated and supervised data collection approved the final manuscript.

All authors approved the final manuscript as submitted and agree to be accountable for all aspects of the work. 


\section{ABSTRACT}

Background: Peanut allergens are common responsible of food allergy. Analyses of sensitization patterns, relationships with other allergens, clinical symptoms and variation with age are needed. We studied sensitization to Ara h 2, Ara h 9 and Pru p 3 in a population of children/adolescents allergic to peanut, and the relation with peach and pollen.

Methods: Patients allergic to peanut were classified into two groups: A) allergic to peanut, and B) allergic to peach and peanut. The IgE response was measured to Ara h 2, Ara h 9 and Pru p 3.

Results: Of 456 subjects evaluated, 28\% were allergic to peanut. From this group 69\% were sensitized to pollen. Urticaria was the most frequent entity followed by anaphylaxis and OAS. Fifty-nine percent had Ara h 2 and/or Ara h 9 specific IgE. Over half reported symptoms with peanut alone (Group A); 46\% to peanut and peach (Group B). We observed significant differences in sex, age, onset of symptoms and sensitization to Artemisia. IgE response to Ara h 2 was more frequent in Group A; Ara h 9 and Pru p 3 in Group B. We observed a decrease in sensitization to Ara h 2 and an increase to Ara h 9 and Pru p 3 with increasing age.

Conclusion: Peanut allergy is frequent in subjects with allergy to plant-foods, with Ara h 2 and Ara h 9 being two important allergens. In younger patients Ara h 2 predominates over Ara h 9. The reverse was observed in older patients. 


\section{INTRODUCTION}

The prevalence of food allergy varies between $1 \%$ and $3 \%$ in the general population and is on the rise (1-4). Immediate reactions to food of plant origin are one of the most frequent causes of food allergy, with many cases starting in infancy $(5,6)$. Peanut allergens are frequently implicated, affecting $1 \%$ of the population (7). Several studies have reported an allergy prevalence rate of $0.50 \%-1.85 \%$ in children $(1,8)$. However, more studies need to be undertaken in other populations and with larger age ranges.

The major peanut allergens Ara h 1, Ara h 2 and Ara h 3 are vicilin, conglutin (2S Albumin) and legumin seed storage proteins (SSP), respectively (9-14). Ara h 8 is a pathogenesis-related protein, PR-10 (15,16); Ara h 9 belongs to the lipid transfer protein (LTP) family, many of which are important allergens in the Mediterranean area (17-19). Many plant allergens are panallergens, present in many different sources. They are responsible for the cross-reactivity between homologous proteins from plant-derived foods and pollens (20-22). In central European countries a strong association exists between pollen sensitisation and food allergy. For example, apple and peach sensitization has been associated with primary sensitization to Bet v 1, the major birch pollen allergen, as well as Mal d 1 or Pru p 1, all belonging to the PR-10 family (23). Other associations between pollen and plant-derived foods have been described, along with details of the responsible allergens $(24,25)$. This situation varies in the Mediterranean area where sensitization to Pru p 3 (LTP from peach) has been shown to be unrelated to pollen allergies $(26,27)$.

Values of specific IgE to single molecular allergens may vary according to age. For example, peanut allergic children and adults have shown differences in allergen molecular recognition, with Ara h 2 the most prevalent in children (28) and Ara h 9 in adults (18). It is known that Ara h 9 sensitization is due to cross reactivity with Pru p 3, 
both LTPs $(19,29)$. However, the reasons for the age-related differences in allergen recognition are complex and cannot be explained in term of allergen exposure and the cross-reactivity phenomenon only. Detailed studies of how these patterns of sensitization occur at different ages in the same population and the relationship with related foods/inhalants are needed. This is particularly relevant in populations where primary sensitization occurs through the oral route.

In order to verify these variations in the molecular response to plant food allergens we undertook a cross-sectional study in a population ranging from 1 to 20 years of age. This provides a clearer view of sensitization patterns in different age groups. Those confirmed as allergic to peanut were further studied in order to verify the role of major peanut and peach molecular allergens in inducing sensitization and symptoms. Results indicate that the predominate allergen differs according to age: Ara $\mathrm{h} 2$ for younger children and Ara h 9 for older children and adolescents. These findings have important clinical consequences. 


\section{MATERIAL AND METHODS}

\section{Subject selection}

All patients were aged between 1 and 20 years old and referred to our service with suspicion of food allergy of plant origin were included in our study. The area of study was Madrid, Central Spain. All participants completed a written informed consent and the ethical committee of our institution approved the study.

The allergological evaluation included an examination of the patient's clinical history with implementation of an exhaustive questionnaire and skin prick test (SPT) as detailed below. In subjects with unclear history, double-blind-placebo-controlled food challenge (DBPCFC) was carried out.

The SPT was performed according to European guidelines (30) using a standardized panel of relevant inhalant allergenic extracts that included Phleum, Olea, Platanus, and Artemisia pollens plus peach and peanut from ALK-Abelló (Madrid, Spain). The SPT response was considered positive if the diameter of the wheal area was $3 \mathrm{~mm}$ greater than that induced by the negative control.

Patients were considered allergic if their clinical history showed at least two convincing reactions in their history within the first hour after exposure. In those with clear clinical history but only one episode to peanut, DBPCFC was performed to confirm the diagnosis. DBPCFC was performed as described previously (31). Briefly, meals containing $5 \mathrm{~g}$ of amount of flour peanut with a mixture of yogurt, orange juice, dried coffee and oatmeal flakes were prepared. Placebo meals consisted of the same ingredients, without peanut. If cutaneous and/or respiratory symptoms or alterations in vital signs appeared, the procedure was stopped and the symptoms were evaluated and treated (32). We excluded subjects with pathologies such as genetic, autoimmune and metabolic diseases. 
Quantification of IgE antibodies to whole peanut and peach extract and allergens

Specific IgE antibodies to whole peanut and peach extract and allergens Ara h 2, Ara h 9 and Pru p 3 were measured by ImmunoCAP following the manufacturer's recommendations (Phadia, Uppsala, Sweden). A positive result was defined as a value > $0.35 \mathrm{kUA} / \mathrm{l}$.

\section{Statistical analysis}

Quantitative variables are shown as median and interquartile ranges (IR), while qualitative variables are shown as frequencies. Medians between groups were compared using Mann-Whitney or Kruskal-Wallis tests, while $\chi^{2}$ was used to compare proportions. Differences with a $\mathrm{p}<0.05$ were considered significant. 


\section{RESULTS}

\section{Characteristics of peanut allergic patients}

We evaluated a population of children and adolescents ranging from 2 to 20 years old who reported symptoms after peanut intake and/or contact. After proving hypersensitivity we studied peanut allergy in the context of sensitization to other relevant allergens including food and inhalants. We established the relationship between them by focusing on three relevant allergens in our population: Ara h 2, Ara h 9 and Pru p 3.

From the total number of 456 cases allergic to plant foods initially evaluated, 28\% (128 cases) of the patients reported symptoms with peanut, 260 showed tolerance to peanut and the remaining 68 subjects had not had previous contact with peanut. Of the 128 patients with reported symptoms, 120 were confirmed as hypersensitive to peanut. Of these, 91 were diagnosed by clinical history and positive skin prick test and 29 by DBPCFC. The remaining 8 cases had good tolerance in spite of reported symptoms after peanut ingestion in their clinical history and were not used for further study. The median age of peanut hypersensitive patients was 9.86 (IR:2-20) years and 55\% were male. Skin test response to inhalant allergens was positive for $96 \%$ of the cases, with $69 \%$ sensitive to pollens. The majority of patients (88\%) had other allergic diseases in addition to food allergy. These were atopic dermatitis (63\%), rhinoconjuntivitis (68\%) and asthma (51\%). The clinical symptoms most frequently reported by the patients was urticaria/angioedema in 58 patients (48\%), followed by anaphylaxis (dyspnea, wheeze and hypotension) in 33 (28\%) and OAS in 29 (24\%).

Detailed analysis of these patients showed that 42 were also allergic to peach. The clinical symptoms reported by these patients were: OAS in 20 patients (48\%), urticaria/angioedema in 18 (42\%) and anaphylaxis in 4 (9\%). 


\section{Skin prick test results}

Most patients (91\%) showed positive skin prick test results to peanut. More detailed analysis of these results showed that $90 \%$ of patients with anaphylaxis were skin test positive to peanut, as were $89 \%$ of patients with urticaria/angioedema. For patients with OAS, $79 \%$ of patients were skin test positive to peanut. Out of the 11 patients with negative SPT, 4 had urticaria/angioedema, 4 had OAS and 3 cases had anaphylaxis. With respect to pollen allergy, $87 \%$ of peanut allergic subjects were positive for Phleum, 76\% for Olea, 73\% for Platanus and 45\% for Artemisia.

\section{Specific IgE results}

From 120 patients studied, 83\% had specific IgE to whole peanut extract. When we measured specific IgE to whole peach extract to patients allergic to peanut and peach we observed that $90 \%$ of these had specific IgE to peach extract.

Measuring IgE response to peanut allergens showed that Ara h 2 antibodies were detected in 33\% of patients and Ara h 9 in 42\% (Figure 1A). Considering cases with specific IgE to only one allergen, 18\% were positive to Ara h 2 and $27 \%$ to Ara h 9 only. Out of all peanut allergic patients tested, 59\% were positive to at least one allergen (Figure 1B).

Of the patients with positive SPT to peanut, 68\% showed IgE antibodies and of the 11 cases with negative SPT, 7\% had IgE antibodies.

The proportion of patients with specific IgE to Ara h 2 and Ara h 9 was similar for patients with anaphylaxis and urticaria. For OAS, a higher proportion of patients showed IgE to Ara h 9 (48\%) compared to Ara h 2 (24\%) (Figure 2).

Comparison between peanut allergic patients with tolerance to peach and patients allergic to both peanut and peach. 
In order to establish the relationship between peanut and peach allergy we compared the results in those patients with allergy to peanut but tolerant to peach (Group $A, N=64$ ) and those with symptoms induced by both peanut and peach (Group B, N=42) (Table 1). Those subjects who never took peach $(\mathrm{N}=14)$ were removed from this analysis. The patients of Group A were younger and the symptoms appeared earlier than in Group B. The overall pollen sensitization in Group A was lower than in Group B, but if we analyzed this response considering the pollens individually we detected that Phleum, Olea and Platanus were similar between both groups, however the percentage of Artemisia was significant higher $(\mathrm{p}=0.033)$ in Group B. Significant differences were observed between groups for patient age, age at symptom onset, sex, IgE specific to peach and sensitization to pollens.

When we compared the clinical symptoms induced, anaphylaxis appeared in $31 \%$ of the cases in Group A and in 19\% of Group B ( $\mathrm{p}=0.014)$, urticaria/angioedema was similar for both (52\% in Group A and 44\% in Group B). OAS was more common in Group B with $37 \%$ compared to $15 \%$ of Group A ( $\mathrm{p}=0.022$ ) (Figure 3).

The IgE response to individual peanut and peach allergens was analysed. When we compared Group A and Group B, we observed the number of individuals with IgE to Ara h 9 and Pru p 3 was significantly higher ( $p=0.001$ and $p=0.008$, respectively) in Group B. Within Group A, Ara h 2 was the most frequent allergen, although this difference did not reach statistical significance. Within Group B Ara h 9 and Pru p 3 were significantly more prevalent than Ara h 2 ( $p=0.003$ and $p=0.012$, respectively) (Figure 4).

When taking into account combinations of allergens we found that for Group A the cases positive to Ara h 2 were the most frequent $(\mathrm{p}=0.050)$ followed by the combination of Ara h 2, Ara h 9 and Pru p 3. Very few cases showed IgE to the combination of Ara h 
9 and Pru p 3 only. On the other hand, for cases with allergy to both peanut and peach, the highest number of positive results corresponded to the combination of Ara h 9 and Pru p 3 ( $p=0.018$ ) only; few cases were found that were positive to Ara h 2 only (Table 2).

\section{Differences in the recognition of peanut and peach allergens according to age}

We grouped patients by age intervals of 5 years from youngest to oldest in order to evaluate differences in the IgE response to Ara h 2, Ara h 9 and Pru p 3 (Figure 5). We observed that values of specific antibodies showed a significant decrease of Ara h 2 allergen prevalence with increasing age (Figure 5A). In contrast, the number of patients with antibodies to Ara h 9 and Pru p 3 increased with age (Figure 5B, 5C). We conclude that Ara h 2 positivity was much more prevalent in young children than Ara h 9 and Pru p 3. However the opposite was observed for older age groups. 


\section{DISCUSSION}

In this work we have performed a cross-sectional study in a large group of children and adolescents with allergy to plant foods in a centre located in central Spain (Madrid). More than a quarter of patients reported symptoms with peanut, in agreement with previous studies in a similar population $(3,34,35)$. We also observed association with other allergic diseases such as atopic dermatitis and asthma, as reported previously (36). Reactions to plant allergens are highly associated with pollen allergy $(20,21,37)$. In our population we observed a variable percentage of sensitization to the pollens tested with Phleum, Olea and Platanus higher that 50\%. Whether this association is related to cross-reactivity or represents a clinical property of atopic patients is a matter of speculation, however all these pollens contain panallergens with similar structures to peanut and peach proteins, such as profilins, PR-10 and LTPs. Focusing on the pollens with LTP allergens, we observed a strong association between patients allergic to both peanut and peach as food sources, and Artemisia pollen, suggesting cross-reactivity across LTP proteins (38-39).

Considering the clinical symptoms, over three quarters of peanut allergic patients observed in the study developed systemic symptoms, and more than a quarter developed anaphylaxis. These findings are similar to those reported by Pedrosa (28), where urticaria/angioedema was the most frequent clinical symptom followed by OAS and anaphylaxis.

The diagnosis of peanut allergy in our study was largely based on an unequivocal history of several episodes after ingestion of peanut. We detected that SPT was positive to peanut in $91 \%$ of these patients and $83 \%$ of them had specific IgE antibodies to peanut. Similar results have been described in other studies (40). For patients with anaphylaxis, 95\% were SPT positive, and 75\% had detectable IgE antibodies to 
allergens peanut. For urticaria/angioedema $89 \%$ were SPT positive and $66 \%$ had detectable IgE antibodies. For those patients with OAS, 88\% had a positive SPT and 76\% had detectable IgE antibodies. Interestingly, we detected IgE antibodies to purified allergens for $7 \%$ of the cases that produced a negative SPT. This result may be due to a low concentration of the relevant allergen in the whole extract used for SPT, as suggested by others (41).

Regarding the sensitization profile to peanut allergens, we found that $42 \%$ of patients had IgE to Ara h 9, and 27\% of the patients were monosensitized to this allergen.

A third of all patients recognized Ara h 2 and 18\% were monosensitized. Our results are in line with a recent study from Calamelli (42) but are lower than those of Vereda et al (19). More than half (59\%) of all patients had IgE antibodies to at least one allergen. Ara h 9 is more prevalent in the population studied here and appears to play an important role in peanut allergy among patients from the Mediterranean area $(17,18)$. Ara h 2 has been implicated in other populations (28,43-45). For example, the work of Vereda et al. (19) showed that Ara h 2 was recognised by $42 \%$ of patients with peanut allergy.

Our study revealed an age dependent sensitization pattern in patients allergic to peanut extract. Interestingly, we found that the youngest group of children were more frequently monosensitized to Ara h 2: $32 \%$ at $1-5$ years and $20 \%$ at 6-10 years. However the opposite pattern was found for Ara h 9: no patients in the 1-5 year group were monosensitized, however for 6-10 year old subject, 36\% were monosensitised. Similar results have been described in a recent study where levels of IgE to Ara h 2, Ara h 8 , Ara h 9 were measured in a pediatric population (42). It seems that natural loss of sensitisation occurs to Ara h 2 with increasing age, while awareness of LTP is gradually beginning. The presence of members of the LTP family in many species could be like a 
maintenance dose of the allergen for these individuals. However, the absence of cross reactivity between $2 \mathrm{~S}$ albumins due to the high difference in the primary structure of these proteins may lead a reduction in the allergic response to the allergen (46).

Regarding the relationship between clinical symptoms and the pattern of allergen recognition, fewer patients with OAS had IgE against Ara h 2 than Ara h 9. However, we did not find any differences for anaphylaxis or urticaria/angioedema. Our data agree with previous studies $(28,47)$, although one study has shown anaphylaxis to be more highly associated with Ara h 2 (48).

The association of peach and peanut allergy is high in the Mediterranean region, due to cross-reactivity between both peanut and peach LTP (49). However this relation is less marked in other countries as described previously (19). So, our population could be divided into two major groups: patients with allergic reactions to peanut and not peach (Group A), and patients with allergic reactions to both peanut and peach (Group B) as described previously $(29,49,50)$. Almost one third of the peanut allergic patients belonged to Group B. Symptoms with peach often appeared before peanut allergy. This may be due a primary sensitization to peach LTP, Pru p 3, as previously reported by our group in an adult population (29). Sensitization to pollen was higher in patients allergic to peanut and peach than in those only allergic to peanut, especially for Artemisia. This could be due to cross-reactivity between pollen and food as described previously $(39,51)$.

Analysis of the sensitization pattern confirmed that allergen recognition differed between groups. In Group A, Ara h 2 was higher than Ara h 9 or Pru p 3 (peanut and peach LTPs). In Group B, Ara h 9 and Pru p 3 were significantly higher. These findings agree with other studies $(17,18,45,47,50)$. However, only $22 \%$ of cases from Group A had systemic reactions (anaphylaxis and urticaria/angioedema) and only 12\% of Ara h 2 
positive cases Ara h 2 from Group B had systemic reactions. In 10\% of cases in Group A, the subject had IgE antibodies to Ara h 2 and Ara h 9. Because these subjects developed symptoms to peanut only and tolerated peach, we conclude that Ara h 2 was responsible for the symptoms.

For some cases in Group B, specific IgE was detected for Ara h 9 but not Pru p 3, indicating that Ara h 9 can be the primary sensitizing agent. Ara h 9 has been described as the primary sensitizer in adults (29).

Summarizing, our study presents the largest series of child and adolescent peanut allergic patients and suggests that the sensitization profile is related to sensitivity to other foods and pollen. Moreover, the proportion of patients with specific IgE antibodies varies according to age: for older children and adolescents, fewer patients show IgE to the Ara h 2, whilst more patients show IgE to the LTP proteins Ara h 9 and Pru p 3. 
ACKNOWLEDGEMENTS

We thank Mirian Franco for technical assistance in the diagnostic studies. 


\section{REFERENCES}

1. Venter C, Hasan Arshad S, Grundy J, et al. Time trends in the prevalence of peanut allergy: three cohorts of children from the same geographical location in the UK. Allergy 2010; 65:103-8.

2. Rona RJ, Keil T, Summers C, et al. The prevalence of food allergy: a metaanalysis. J Allergy Clin Immunol 2007; 120:638-46.

3. Young E, Stoneham MD, Petruckevitch A, et al. A population study of food intolerance. Lancet 1994; 343:1127-30.

4. Kaya A, Erkocoglu M, Civelek E, et al. Prevalence of confirmed IgE-mediated food allergy among adolescents in Turkey. Pediatr Allergy Immunol 2013; 24:456-62.

5. Fernandez Rivas M. Food allergy in Alergologica-2005. J Investig Allergol Clin Immunol 2009; 19 Suppl 2:37-44.

6. Zuidmeer L, Goldhahn K, Rona RJ, et al. The prevalence of plant food allergies: a systematic review. J Allergy Clin Immunol 2008; 121:1210-8 e4.

7. Nicolaou N, Poorafshar M, Murray C, et al. Allergy or tolerance in children sensitized to peanut: prevalence and differentiation using component-resolved diagnostics. J Allergy Clin Immunol 2010; 125: 191-7 e1-13.

8. $\quad$ Sampson HA. 9. Food allergy. J Allergy Clin Immunol 2003; 111:S540-7.

9. Burks AW, Williams LW, Connaughton $\mathrm{C}$, et al. Identification and characterization of a second major peanut allergen, Ara h II, with use of the sera of patients with atopic dermatitis and positive peanut challenge. J Allergy Clin Immunol 1992; 90:962-9.

10. Burks AW, Cockrell G, Stanley JS, et al. Recombinant peanut allergen Ara h I expression and IgE binding in patients with peanut hypersensitivity. J Clin Invest 1995; 96:1715-21.

11. de Jong EC, Van Zijverden M, Spanhaak S, et al. Identification and partial characterization of multiple major allergens in peanut proteins. Clin Exp Allergy 1998; 28:743-51.

12. Kleber-Janke T, Crameri R, Appenzeller U, et al. Selective cloning of peanut allergens, including profilin and $2 \mathrm{~S}$ albumins, by phage display technology. Int Arch Allergy Immunol 1999; 119:265-74.

13. Eigenmann PA, Burks AW, Bannon GA, et al. Identification of unique peanut and soy allergens in sera adsorbed with cross-reacting antibodies. J Allergy Clin Immunol 1996; 98:969-78.

14. Radauer C, Bublin M, Wagner S, et al. Allergens are distributed into few protein families and possess a restricted number of biochemical functions. J Allergy Clin Immunol 2008; 121:847-52 e7.

15. Mittag D, Akkerdaas J, Ballmer-Weber BK, et al. Ara h 8, a Bet v 1homologous allergen from peanut, is a major allergen in patients with combined birch pollen and peanut allergy. J Allergy Clin Immunol 2004; 114:1410-7.

16. Asarnoj A, Ostblom E, Ahlstedt S, et al. Reported symptoms to peanut between 4 and 8 years among children sensitized to peanut and birch pollen - results from the BAMSE birth cohort. Allergy 2010; 65:213-9.

17. Lauer I, Dueringer N, Pokoj S, et al. The non-specific lipid transfer protein, Ara h 9, is an important allergen in peanut. Clin Exp Allergy 2009; 39:1427-37.

18. Krause S, Reese G, Randow S, et al. Lipid transfer protein (Ara h 9) as a new peanut allergen relevant for a Mediterranean allergic population. J Allergy Clin Immunol 2009; 124:771-8 e5. 
19. Vereda A, van Hage M, Ahlstedt S, et al. Peanut allergy: Clinical and immunologic differences among patients from 3 different geographic regions. J Allergy Clin Immunol 2011; 127:603-7.

20. Caballero T, Martin-Esteban M. Association between pollen hypersensitivity and edible vegetable allergy: a review. J Investig Allergol Clin Immunol 1998; 8:6-16.

21. Eriksson NE, Formgren H, Svenonius E. Food hypersensitivity in patients with pollen allergy. Allergy 1982; 37:437-43.

22. Mortz CG, Andersen KE, Bindslev-Jensen C. The prevalence of peanut sensitization and the association to pollen sensitization in a cohort of unselected adolescents--The Odense Adolescence Cohort Study on Atopic Diseases and Dermatitis (TOACS). Pediatr Allergy Immunol 2005; 16:501-6.

23. Vanek-Krebitz M, Hoffman-Sommergruber $K$, Laimer da Camara $M$ et al. Cloning and sequencing of Mal d 1, the major allergen from apple (Malus domestica), and its immunological relationship to Bet v 1, the major birch pollen allergen. Biochem Biophys Res Commun. 1995;214(2):538-51.

24. Niggemann B, Schmitz R, Schlaud $M$. The high prevalence of peanut sensitization in childhood is due to cross-reactivity to pollen. Allergy 2011; 66:980-1.

25. Egger M, Mutschlechner S, Wopfner N, et al. Pollen-food syndromes associated with weed pollinosis: an update from the molecular point of view. Allergy 2006; 61:461-76.

26. Fernandez-Rivas M, van Ree R, Cuevas M. Allergy to Rosaceae fruits without related pollinosis. J Allergy Clin Immunol 1997; 100:728-33.

27. Fernandez-Rivas M, Bolhaar S, Gonzalez-Mancebo E, et al. Apple allergy across Europe: how allergen sensitization profiles determine the clinical expression of allergies to plant foods. J Allergy Clin Immunol 2006; 118:481-8.

28. Pedrosa M, Boyano-Martinez T, Garcia-Ara MC, et al. Peanut seed storage proteins are responsible for clinical reactivity in Spanish peanut-allergic children. Pediatr Allergy Immunol 2012; 23:654-9.

29. Javaloyes G, Goikoetxea MJ, Garcia Nunez I, et al. Pru p 3 acts as a strong sensitizer for peanut allergy in Spain. J Allergy Clin Immunol 2012; 130:1432-4 e3.

30. Dreborg S. Skin tests in the diagnosis of food allergy. Pediatr Allergy Immunol 1995; 6 Suppl 8:38-43.

31. Vlieg-Boerstra BJ, Bijleveld CM, van der Heide S, et al. Development and validation of challenge materials for double-blind, placebo-controlled food challenges in children. J Allergy Clin Immunol 2004; 113:341-6.

32. Fleischer DM, Burks AW, Vickery BP, et al. Sublingual immunotherapy for peanut allergy: a randomized, double-blind, placebo-controlled multicenter trial. J Allergy Clin Immunol 2013; 131:119-27 e1-7.

33. Diaz-Perales A, Lombardero M, Sanchez-Monge R, et al. Lipid-transfer proteins as potential plant panallergens: cross-reactivity among proteins of Artemisia pollen, Castanea nut and Rosaceae fruits, with different IgE-binding capacities. Clin Exp Allergy 2000; 30:1403-10.

34. Bjornsson E, Janson C, Plaschke P, et al. Prevalence of sensitization to food allergens in adult Swedes. Ann Allergy Asthma Immunol 1996; 77:327-32.

35. Bock SA, Atkins FM. The natural history of peanut allergy. J Allergy Clin Immunol 1989; 83:900-4.

36. Burks W. Skin manifestations of food allergy. Pediatrics 2003; 111:1617-24. 
37. Dreborg S. Food allergy in pollen-sensitive patients. Ann Allergy 1988; 61:41-6.

38. Lauer I, Miguel-Moncin MS, Abel T, $t$ al. Identification of a plane pollen lipid transfer protein (Pla a 3) and its immunological relation to the peach lipidtransfer protein, Pru p 3. Clin Exp Allergy 2007; 37:261-9.

39. Sanchez-Lopez J, Tordesillas L, Pascal M, et al. Role of Art v 3 in pollinosis of patients allergic to Pru p 3. J Allergy Clin Immunol 2014; 133:1018-25.

40. Hill DJ, Heine RG, Hosking CS. The diagnostic value of skin prick testing in children with food allergy. Pediatr Allergy Immunol 2004; 15:435-41.

41. Khan FM, Ueno-Yamanouchi A, Serushago B, et al. Basophil activation test compared to skin prick test and fluorescence enzyme immunoassay for aeroallergen-specific Immunoglobulin-E. Allergy Asthma Clin Immunol 2012; 8:1.

42. Calamelli E, Caffarelli C, Ricci G. Peanut Sensitization Profiles in Italian Children and Adolescents with Specific IgE to Peanuts. Biomed Res Int; 2013:170452.

43. Koppelman SJ, Wensing M, Ertmann M, et al. Relevance of Ara h1, Ara h2 and Ara h3 in peanut-allergic patients, as determined by immunoglobulin E Western blotting, basophil-histamine release and intracutaneous testing: Ara h2 is the most important peanut allergen. Clin Exp Allergy 2004; 34:583-90.

44. Asarnoj A, Moverare R, Ostblom E, et al. IgE to peanut allergen components: relation to peanut symptoms and pollen sensitization in 8-year-olds. Allergy 2010; 65:1189-95.

45. Nicolaou N, Murray C, Belgrave D,et al.. Quantification of specific IgE to whole peanut extract and peanut components in prediction of peanut allergy. $\mathrm{J}$ Allergy Clin Immunol 2011; 127:684-5.

46. Barre A, Borges JP, Culerrier R, et al. Homology modelling of the major peanut allergen Ara h 2 and surface mapping of IgE-binding epitopes. Immunol Lett 2005; 100:153-8.

47. Flinterman AE, van Hoffen E, den Hartog Jager CF, et al. Children with peanut allergy recognize predominantly Ara h2 and Ara h6, which remains stable over time. Clin Exp Allergy 2007; 37:1221-8.

48. Kulis M, Chen X, Lew J, et al. The 2S albumin allergens of Arachis hypogaea, Ara h 2 and Ara h 6, are the major elicitors of anaphylaxis and can effectively desensitize peanut-allergic mice. Clin Exp Allergy 2012; 42:326-36.

49. Romano A, Fernandez-Rivas M, Caringi M, et al. Allergy to peanut lipid transfer protein (LTP): frequency and cross-reactivity between peanut and peach LTP. Eur Ann Allergy Clin Immunol 2009; 41:106-11.

50. Mayorga C, Gomez F, Aranda A, et al. Basophil response to peanut allergens in Mediterranean peanut-allergic patients. Allergy. 2014; 69:964-8.

51. Cuesta-Herranz J, Lazaro M, Martinez A, et al. Pollen allergy in peach-allergic patients: sensitization and cross-reactivity to taxonomically unrelated pollens. J Allergy Clin Immunol 1999; 104:688-94. 
Table 1. Comparison of demographic and clinical characteristics of patients with symptoms to peanut and tolerance to peach (Group A) and patients with symptoms to peanut and peach (Group B). Significant differences $(\mathrm{P}<0.05)$ are indicated in bold.

\begin{tabular}{|c|c|c|c|}
\hline & $\begin{array}{l}\text { SYMPTOMS TO } \\
\text { PEANUT AND } \\
\text { TOLERANCE TO } \\
\text { PEACH } \\
(\mathrm{N}=64)\end{array}$ & $\begin{array}{c}\text { SYMPTOMS TO } \\
\text { PEANUT AND } \\
\text { PEACH } \\
(\mathbf{N}=42)\end{array}$ & $\mathbf{P}$ \\
\hline Age, median (IR) & $8.77(2-20)$ & $11.87(2-20)$ & 0.010 \\
\hline $\begin{array}{l}\text { Age at onset of symptoms, } \\
\text { median (IR) }\end{array}$ & $5.71(1-17)$ & $\begin{array}{l}\text { Peanut: } 8.45 \text { (1-19) } \\
\text { Peach: } 6.68 \text { (1-19) }\end{array}$ & 0.016 \\
\hline Sex, \% Male & 63 & 38 & 0.020 \\
\hline Caucasian, \% & 86 & 91 & 0.559 \\
\hline $\begin{array}{l}\text { Specific IgE, \% } \\
\text { Peanut whole extract } \\
\text { Peach whole extract }\end{array}$ & $\begin{array}{l}80 \\
61\end{array}$ & $\begin{array}{l}93 \\
93\end{array}$ & $\begin{array}{l}0.145 \\
\mathbf{0 . 0 0 3}\end{array}$ \\
\hline Atopy, \% & 95 & 84 & 0.324 \\
\hline $\begin{array}{l}\text { Sensitization to pollen, \% } \\
\text { Phleum } \\
\text { Olea } \\
\text { Platanus } \\
\text { Artemisia }\end{array}$ & $\begin{array}{l}61 \\
93 \\
70 \\
73 \\
33\end{array}$ & $\begin{array}{l}84 \\
82 \\
82 \\
79 \\
57\end{array}$ & $\begin{array}{l}\mathbf{0 . 0 3 1} \\
0.156 \\
0.301 \\
0.660 \\
\mathbf{0 . 0 3 3}\end{array}$ \\
\hline $\begin{array}{l}\text { Associated Allergic } \\
\text { Diseases, \% }\end{array}$ & 86 & 91 & 0.559 \\
\hline
\end{tabular}


Table 2. Comparison of percentages of positive cases to single or to combinations of allergens Ara h 2, Ara h 9 and Pru p 3 for patients with symptoms to peanut and tolerance to peach (Group A) and patients with symptoms to peanut and peach (Group B). Significant differences $(\mathrm{P}<0.05)$ are indicated in bold.

\begin{tabular}{|c|c|c|c|}
\hline & $\begin{array}{c}\text { SYMPTOMS TO } \\
\text { PEANUT AND } \\
\text { TOLERANCE } \\
\text { TO PEACH } \\
\mathbf{( N = 6 4 )}\end{array}$ & $\begin{array}{c}\text { SYMPTOMS TO } \\
\text { PEANUT AND } \\
\text { PEACH } \\
\text { (N=42) }\end{array}$ & p \\
\hline Ara h 2 & $20 \%$ & $9 \%$ & 0.050 \\
\hline Ara h 9 & $2 \%$ & $6 \%$ & 0.564 \\
\hline Pru p 3 & $0 \%$ & $3 \%$ & 1 \\
\hline Ara h 9 + Pru p 3 & $6 \%$ & $44 \%$ & $\mathbf{0 . 0 1 8}$ \\
\hline Ara h 2 + Ara h 9 & $2 \%$ & $3 \%$ & 1 \\
\hline Ara h 2 + Pru p 3 & $2 \%$ & $0 \%$ & 1 \\
\hline Ara h 2 + Ara h 9 + Pru p 3 & $16 \%$ & $9 \%$ & 0.132 \\
\hline
\end{tabular}




\section{FIGURE LEGENDS}

Figure 1.- Percentage of positive cases to peanut allergens (Ara h 2 and Ara h 9) in the population reporting symptoms to peanut (measured by ImmunoCAP). A) IgE response to peanut allergens Ara h 2 and Ara h 9. B) IgE response to single peanut allergens (Ara h $2+$ Ara h 9 - , Arah 2 - Arah $9+$ ) and to any or both allergens (Ara h $2+$ and/or Ara h $9+)$.

Figure 2.- Percentage of positive cases to peanut allergens (Ara h 2 and Ara h 9) for patients presenting different clinical symptoms in the population reporting symptoms to peanut (measured by ImmunoCAP).

Figure 3.- Differences between clinical symptoms developed by ingestion and/or contact to peanut in patients with symptoms to peanut and tolerance to peach (Group A : $\mathrm{N}=64$ ) and patients with symptoms to peanut and peach (Group $\mathrm{B}: \mathrm{N}=42$ ). $\mathrm{P}<0.05$ represents a significant difference.

Figure 4.- Percentage of positive cases to peanut and peach allergens (Ara h 2, Ara h 9 and Pru p 3) in patients with symptoms to peanut and tolerance to peach (Group A : $\mathrm{N}=64$ ) and patients with symptoms to peanut and peach (Group $\mathrm{B}: \mathrm{N}=42$ ). $\mathrm{P}<0.05$ represents a significant difference.

Figure 5.- Percentage of positive cases showing specific IgE $(\mathrm{A}, \mathrm{B}, \mathrm{C})$ to peanut and peach allergens (Ara h 2, Ara h 9 and Pru p 3) in patients, grouped by age intervals of 5 years from youngest to oldest. $\mathrm{P}<0.05$ represents a significant difference. 
FIGURE 1

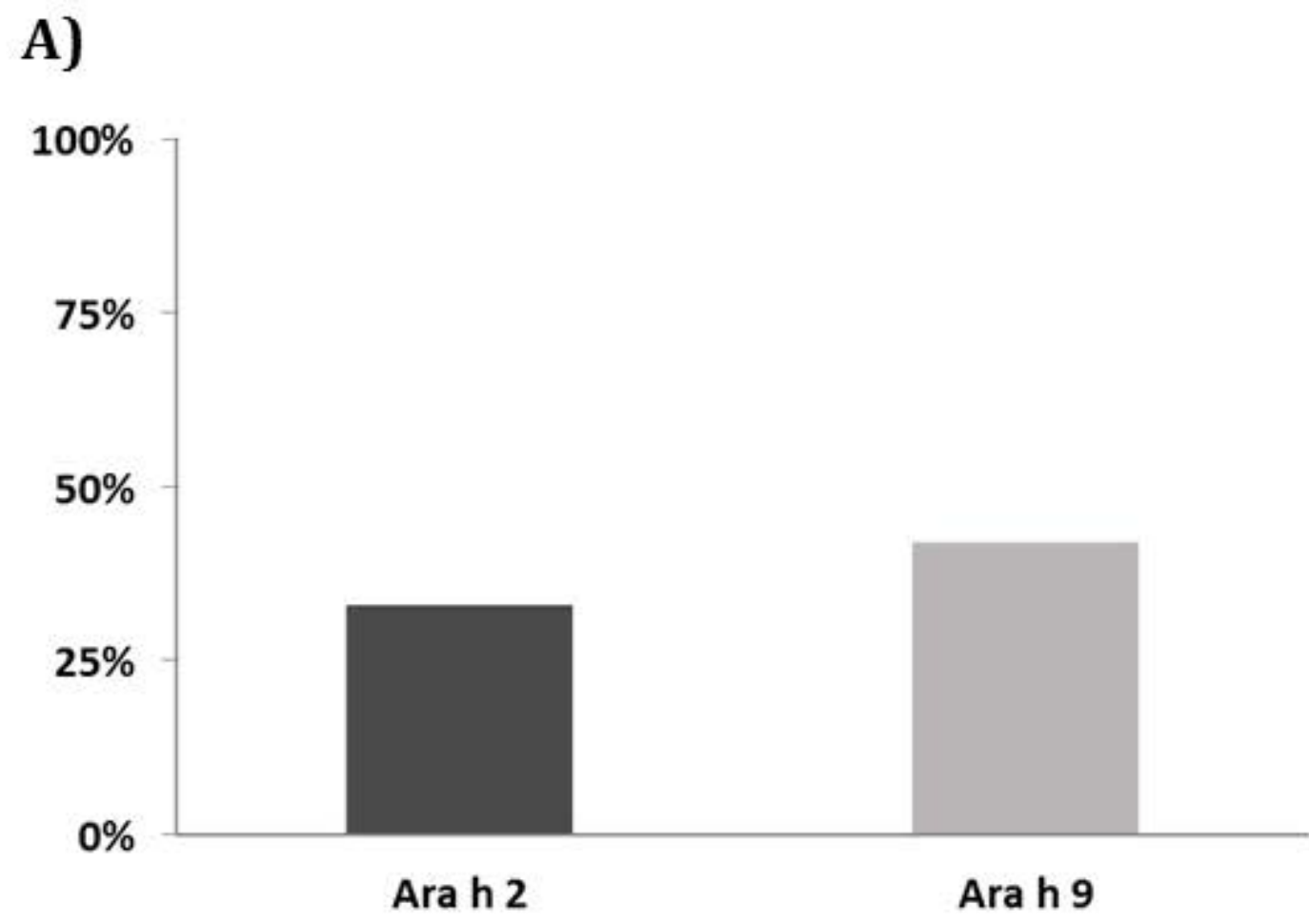

\section{B)}

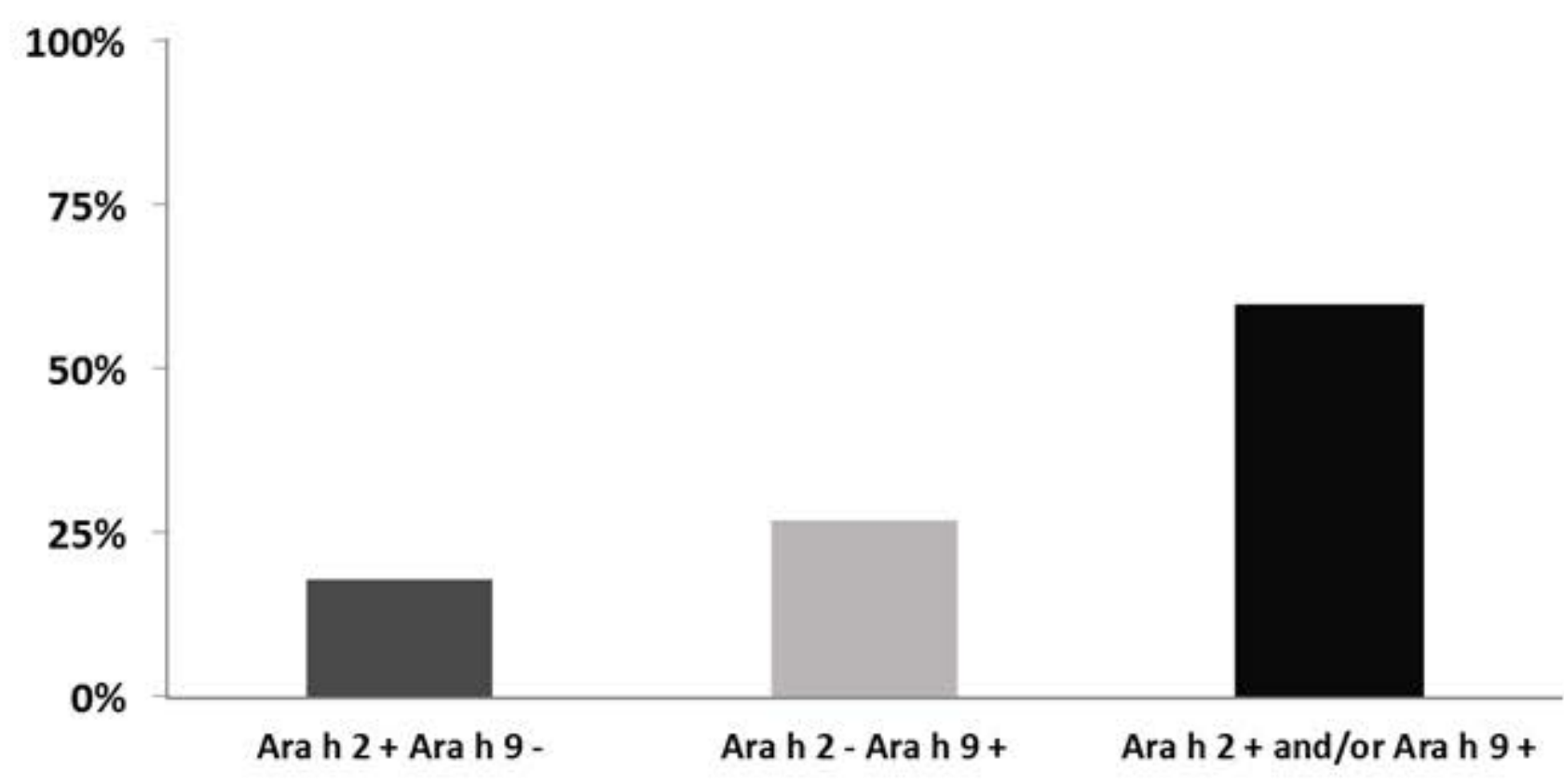




\section{FIGURE 2}

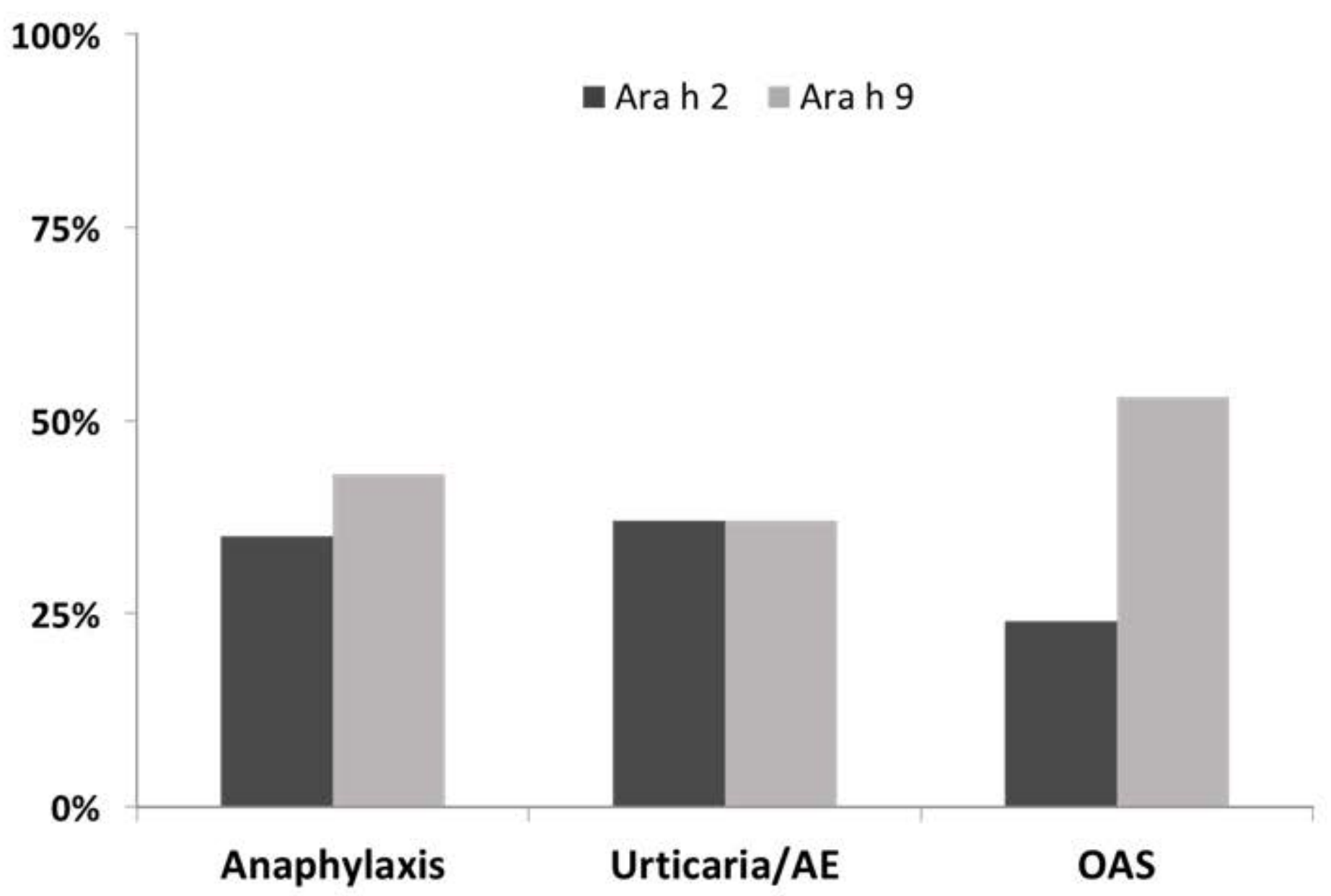




\section{FIGURE 3}

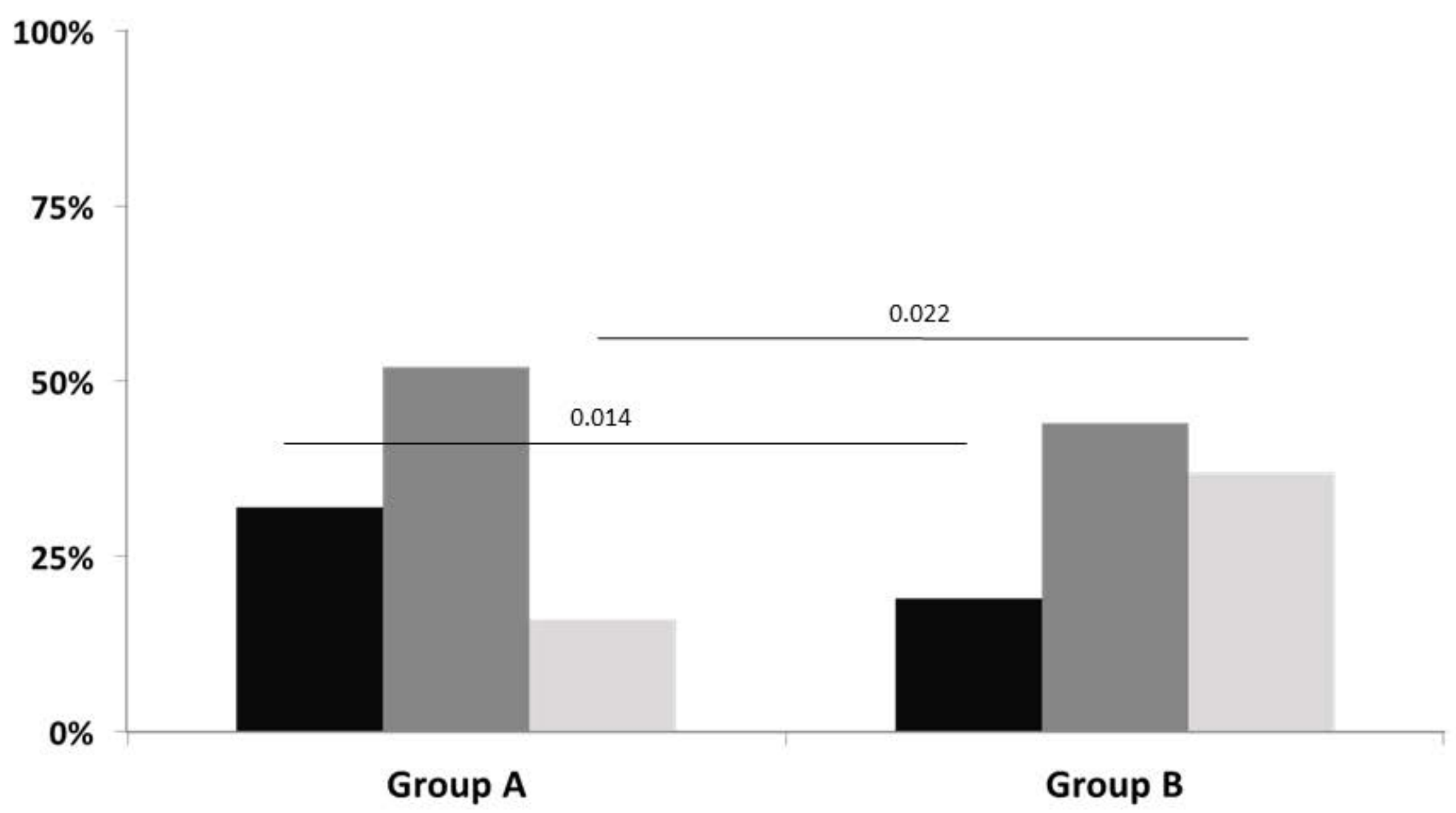

- Anaphylaxis Urticaria/AE 


\section{FIGURE 4}

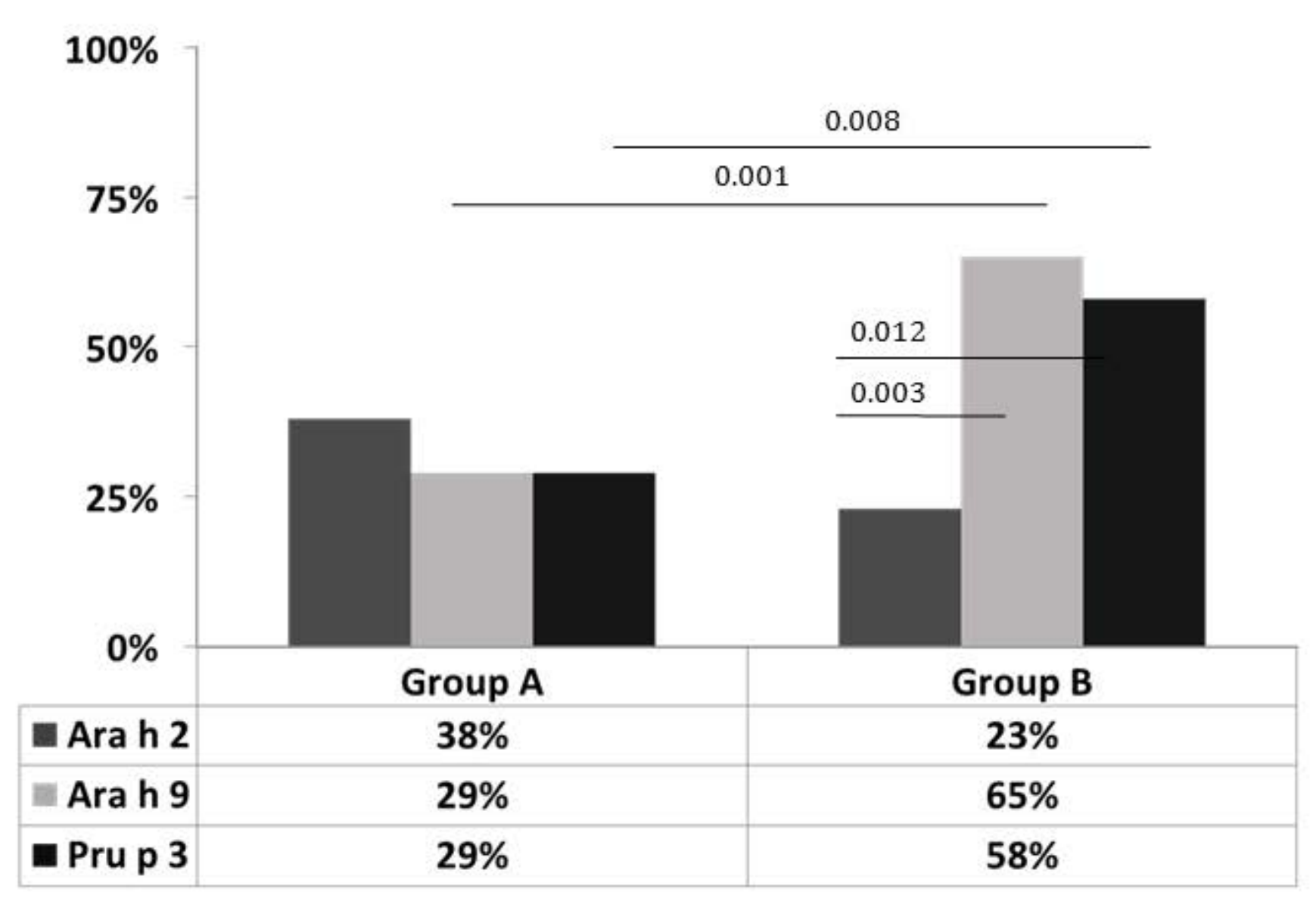




\section{FIGURE 5}

A)

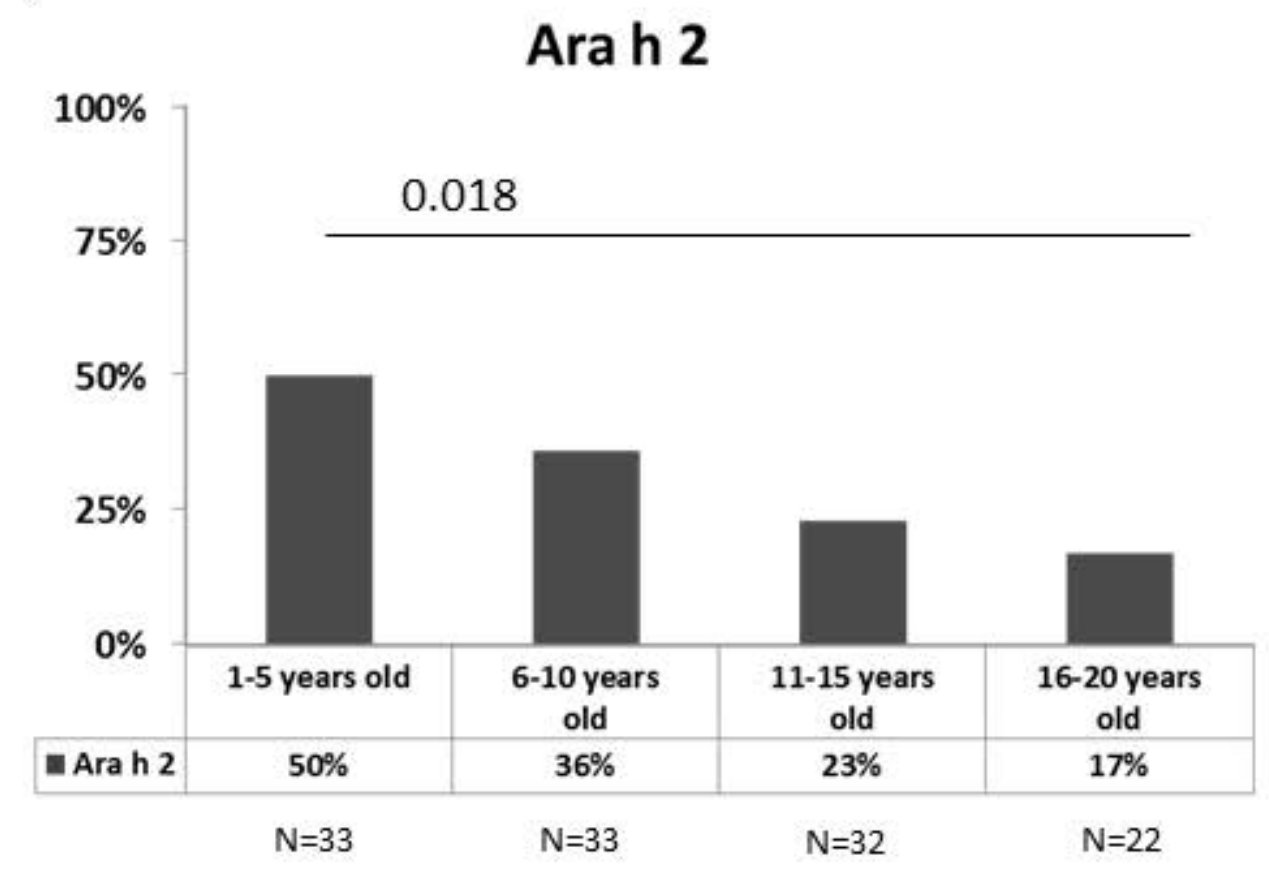

B)

Ara $\mathrm{h} 9$

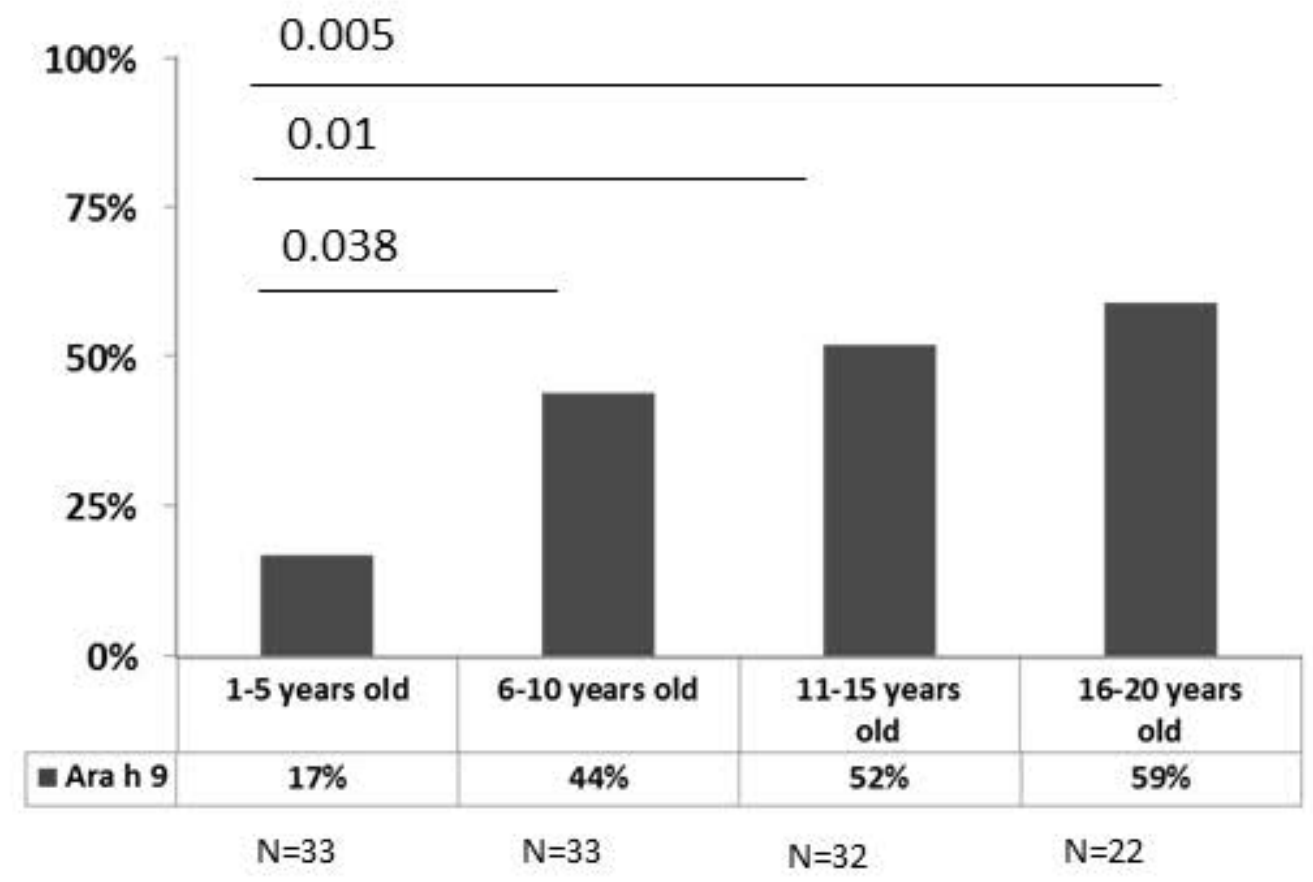

C)

Prup 3

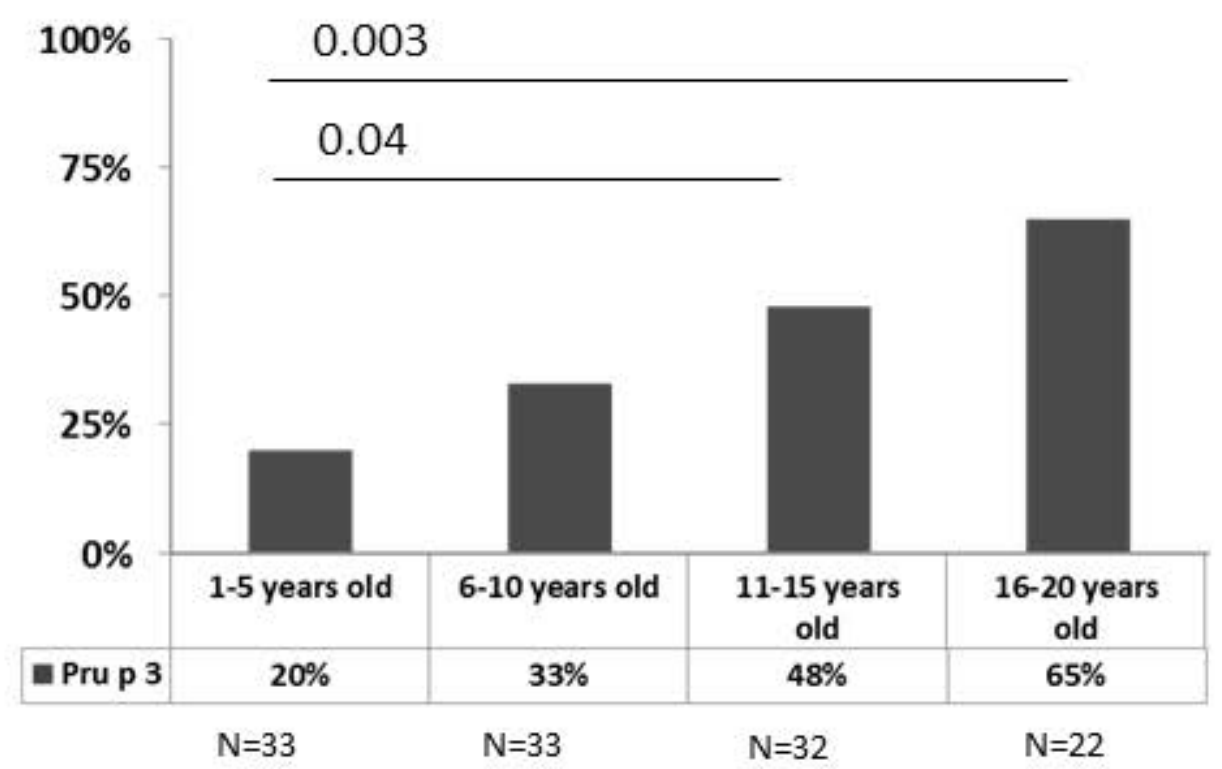

